\title{
Tomates orgânicos: caracterização e vida útil
}

Eduarda Caetano Peixoto, Bianca Pio Ávila, Irajá Ferreira Antunes, Márcia Arocha Gularte

https://doi.org/10.4322/mp.978-65-991393-5-2.c8

\section{Resumo}

O tomate (Solanum lycopersicum L.) é considerado um fruto de sabor agradável e um alimento com muitos benefícios à saúde, é uma das hortaliças mais consumidas no mundo inteiro, seu consumo apresenta crescimento expressivo ao longo dos anos. Diante disso, o objetivo do presente trabalho foi avaliar a vida útil de sete acessos de tomates, oriundos de sistema orgânico, cultivados na Embrapa Terras Baixas na cidade Capão do Leão/RS, armazenados em temperatura ambiente e em temperatura de refrigeração, perante análise sensorial descritiva. Também foram realizadas análises físico-químicas e caracterização de compostos bioativos. As características físico-químicas avaliadas foram $\mathrm{pH}$, teor de sólidos solúveis totais (SST) e cor. Os compostos bioativos avaliados foram: capacidade antioxidante, vitamina $\mathrm{C}$, compostos fenólicos e carotenoides. A amostra número 4 foi a que apresentou pH mais ácido, a amostra número 14 apontou valor alto de SST, sendo a mais doce dentre as amostras analisadas. A casca com coloração mais vermelha foi da amostra número 12. A amostra número 18 apresentou maior capacidade antioxidante, em relação à vitamina $\mathrm{C}$, a amostra número 4 obteve maior teor. A amostra de número 4 teve maior resultado para a quantidade de compostos fenólicos, e a amostra número 6/7 obteve maior quantidade de beta caroteno. As amostras de número 12, 13 e 6/7 mostraram maior vida útil (12 dias em temperatura de refrigeração). O estudo aponta que os acessos podem ser produzidos para o consumo.

Palavras-chave: Solanum lycopersicu L, análise sensorial, antioxidantes.

\section{Introdução}

O consumo de hortaliças tem aumentado devido à maior conscientização da população em busca de uma dieta alimentar mais rica e saudável, o tomate (Solanum lycopersicum L.) é uma hortaliça cultivada e consumida em todo o mundo (COSTA et al., 2010). Segundo o Instituto Brasileiro de Geografia e Estatística o Brasil está, hoje, entre os dez maiores produtores de tomate do mundo, e o estado que mais produz o fruto é Goiás, em 2017 foram produzidos mais de 677 milhões de toneladas (IBGE, 2017). O tomate é ótima fonte de vitaminas A e C e de sais minerais, e pode ser destinado a agroindústria para consumo à mesa. São consumidos na forma de molhos prontos e caseiros, extrato, doces, sucos e cru, na salada. Apesar de ser proibido pela legislação, muitos frutos são comercializados em caixas de madeira, entretanto o ideal é a venda a granel em caixas de plástico, bandejas plásticas ou de isopor, cobertas com filme plástico (GIULIO, 2007). 
O aumento na exigência dos consumidores por alimentos de qualidade e sem produtos químicos, e a busca por uma agricultura ambientalmente sustentável promoveu o desenvolvimento da produção de alimentos orgânicos. Neste sistema de produção existe a preocupação com o uso do solo e com a saúde do consumidor. Muitos agrotóxicos são classificados como extremamente tóxicos para a saúde humana, podendo, em longo prazo, causar diferentes tipos de doenças (VALENT, 2014). O consumo de hortaliças orgânicas no Brasil é muito baixo comparado com outros países. Vários fatores colaboram para que isso aconteça, dentre os quais os custos com a certificação. Muitos produtos orgânicos possuem certificação, é uma forma de controlar a procedência do produto e a diferença na produção em relação à agricultura convencional (NOGUEIRA; ROSADO; GOMES, 2009).

Entretanto, muitos agricultores estão, aos poucos, tentando se adaptar ao processo. Segundo a Instrução Normativa n. 46 06/10/2011 do Ministério da Agricultura Pecuária e Abastecimento (MAPA, 2011), as sementes crioulas utilizadas no cultivo orgânico deverão ser oriundas de sistemas orgânicos e, não existindo no mercado estas sementes adequadas a determinada situação ecológica específica, o produtor poderá lançar mão de produtos existentes no mercado, desde que avaliadas pela instituição certificadora, excluindo-se todos os organismos geneticamente modificados, e o uso de agrotóxico sintético no tratamento e armazenamento de sementes.

\section{Materiais e Métodos}

Foram obtidos tomates de sete acessos oriundos de material crioulo cultivado em sistema orgânico, cedidos pela Embrapa Terras Baixas no município de Capão do Leão, os quais foram classificados em tomate tipo cereja (Tomate 12, 13, 14, 4, e 6/7) e tomate tipo salada (Tomate 16 e 18). Após a colheita, os frutos foram selecionados e higienizados em seguida foram transportados em sacos plásticos para o laboratório de análise sensorial da Universidade Federal de Pelotas. Realizaram-se análises físicoquímicas, $\mathrm{pH}$, sólidos solúveis totais e cor. Também foram feitas análises de compostos bioativos, atividade antioxidante - DPPH, fenóis, vitamina C e carotenoides (estas análises foram feitas apenas no dia da colheita). Para avaliar a vida útil dos tomates foi realizada análise sensorial através de método descritivo.

\subsection{Análises físico-químicas}

\subsubsection{Análise de cor}

A cor das amostras foi determinada através do sistema Cielab com a leitura de três parâmetros, proposto pela Comission Internacionale de l'Eclairage (CIE), que permite medir a intensidade de absorção na região visível para obtenção dos parâmetros $L^{*}, a^{*}$ e $b^{*}$. As análises foram feitas em triplicatas, realizadas com auxílio do colorímetro da marca Minolta ${ }^{\circledR}$, modelo CR-300, em que $L^{*}$ expressa a luminosidade ( $L^{*}=0$ preto e $L^{*}=100$ branco) e $a^{*}$ e $b^{*}$ relacionados acromaticidade $\left(+a^{*}=\right.$ vermelho e $-a^{*}=$ verde; $+b^{\star}=$ amarelo $e-b^{\star}=$ azul).

\subsubsection{Análise de $\mathrm{pH}$}

O pH foi determinado por um medidor digital da marca Hanna ${ }^{\circledR}$ modelo HI 2221. Pesou-se aproximadamente $3 \mathrm{~g}$ de amostra e homogeneizou-se com $5 \mathrm{~mL}$ de água destilada, em seguida foi feita leitura. 


\subsubsection{Sólidos Solúveis Totais (SST)}

Foi obtido através de refratômetro digital de mão da marca ATAGO ${ }^{\circledR}$, modelo PR- 32a, que consiste em medir o índice de refração da amostra, e o resultado foi expresso em ${ }^{\circ}$ Brix.

\subsection{Caracterização de compostos bioativos}

\subsubsection{Vitamina C (ácido L-ascórbico)}

Aproximadamente $2 \mathrm{~g}$ de amostra foi homogeneizada com $10 \mathrm{~mL}$ de solução ácido metafosfórico $(4,5 \%)$ em água ultra pura e deixada em repouso por cerca de 1 hora em frasco protegido da luz. Transferiu-se este volume para um balão de $25 \mathrm{~mL}$ e completou-se o volume com água ultrapura. Filtrou-se a amostra em papel filtro, cuidando para a retirada de toda a amostra, deixando somente o resíduo da filtragem. O sobrenadante da filtragem foi centrifugado em tubos Eppendorf nas condições de 7000 rpm por $10 \mathrm{~min}$. O sobrenadante da centrifugação está pronto para ser analisado em cromatógrafo de alta performance (HPLC), metodologia descrita por Vinci (1995). O conteúdo de vitamina $C$ dos frutos foi determinada através da quantificação da área dos picos gerados pelo cromatógrafo em comparação com a curva padrão de ácido ascórbico e os resultados expressos em $\mathrm{mg} .100 \mathrm{~g}^{-1}$ de fruto fresco.

\subsubsection{Atividade Antioxidante}

Determinada através de espectrofotometria segundo metodologia adaptada de BrandWilliams, Cuvelier e Berset (1995). Esse método é baseado na captura do radical livre DPPH (2,2- difenil-1-picril-hidrazil) por antioxidantes, produzindo um decréscimo da absorbância a $515 \mathrm{~nm}$. A extração foi realizada utilizando $5 \mathrm{~g}$ de fruta adicionadas de $20 \mathrm{~mL}$ de metanol, $100 \mu \mathrm{L}$ de amostra com uma alíquota de $3 \mathrm{~mL}$ de solução de DPPH em tubo de $15 \mathrm{~mL}$, com fundo cônico (Falcon). Os extratos foram armazenados no escuro, por 24 horas, quando se realizou a leitura a $515 \mathrm{~nm}$ em espectrofotômetro. Resultados expressos em mg.TEAC $\mathrm{g}^{-1}$ de fruto fresco.

\subsubsection{Carotenoides}

Aproximadamente $2 \mathrm{~g}$ de amostra foram homogeneizadas com aproximadamente 20 $\mathrm{mL}$ de acetona gelada com auxilio do vortex durante $5 \mathrm{~min}$. Após a filtragem em funil de Buchner, forrado com um papel filtro, o resíduo foi lavado com acetona até que o mesmo ficasse incolor. O filtrado foi transferido para o funil de separação, adicionando- se éter de petróleo $15 \mathrm{~mL}$ e água destilada, promovendo a separação de fases. Descartou-se a fase inferior e continuou-se lavando com água destilada (aproximadamente 4 ou 5 lavagens) até a remoção total da acetona. Transferiu-se 0 pigmento (extrato) para um balão volumétrico $(25 \mathrm{~mL})$ com o auxilio de um funil contendo algodão, no qual são colocadas algumas gramas de sulfato de sódio anidro. Completou-se o balão com éter de petróleo e realizou-se a leitura em espectrofotômetro. Para esse método, utilizou-se a metodologia descrita por Rodriguez-Amaya (2001).

\subsubsection{Fenóis}

Aproximadamente $1 \mathrm{~g}$ de fruto homogeneizado foi transferido para um balão volumétrico de $100 \mathrm{~mL}$, adicionado $60 \mathrm{~mL}$ de água ultrapura e $5 \mathrm{~mL}$ de Reagente de 
Folin-Ciocalteau. Após 8 minutos adicionou-se $20 \mathrm{~mL}$ de $\mathrm{Na}_{2} \mathrm{CO}_{3} 20 \%$ e elevou-se 0 volume para $100 \mathrm{~mL}$ com água ultrapura.

Após repouso de 2 horas ao abrigo da luz a amostra foi filtrada e submetida à leitura espectrofotométrica $(725 \mathrm{~nm})$. A prova em branco consistiu em $1 \mathrm{~mL}$ de água ultrapura em substituição a $1 \mathrm{~g}$ de fruta. Os resultados foram expressos em equivalência de $\mathrm{mg}$ de GAE. $g^{-1}$ de fruto fresco, através de uma curva de calibração. Para esse método, utilizou-se a metodologia descrita por Singleton e Rossi (1965).

\subsection{Análise sensorial}

Para verificação da vida útil, foram utilizados tomates recém colhidos, em que foram avaliados tempos e temperaturas diferentes, tempo zero (tempo inicial, mesmo dia da colheita), oito dias armazenado em temperatura ambiente $\left(25 \pm 1^{\circ} \mathrm{C}\right)$, oito e doze dias armazenado em temperatura de $\left(4 \pm 1{ }^{\circ} \mathrm{C}\right)$. As amostras ficaram armazenadas na geladeira acondicionadas na gaveta de verduras.

Utilizou-se o método de análise descritiva (GULARTE, 2009) para avaliar as características do fruto ao longo do tempo de armazenamento. A análise sensorial foi realizada no Laboratório de Análise Sensorial da Universidade Federal de Pelotas, sendo aprovada pelo Comitê de Ética em Pesquisa. Todos os participantes assinaram o termo de consentimento livre e esclarecido e foram informados sobre os procedimentos do estudo. A amostra, codificada com três dígitos aleatórios, foi oferecida em porções de $10 \mathrm{~g}$ servidas em pratos de porcelana à temperatura ambiente. O teste foi realizado em cabines individuais com luz branca, sem ruído e sem cheiro, e em um momento distante das refeições principais.

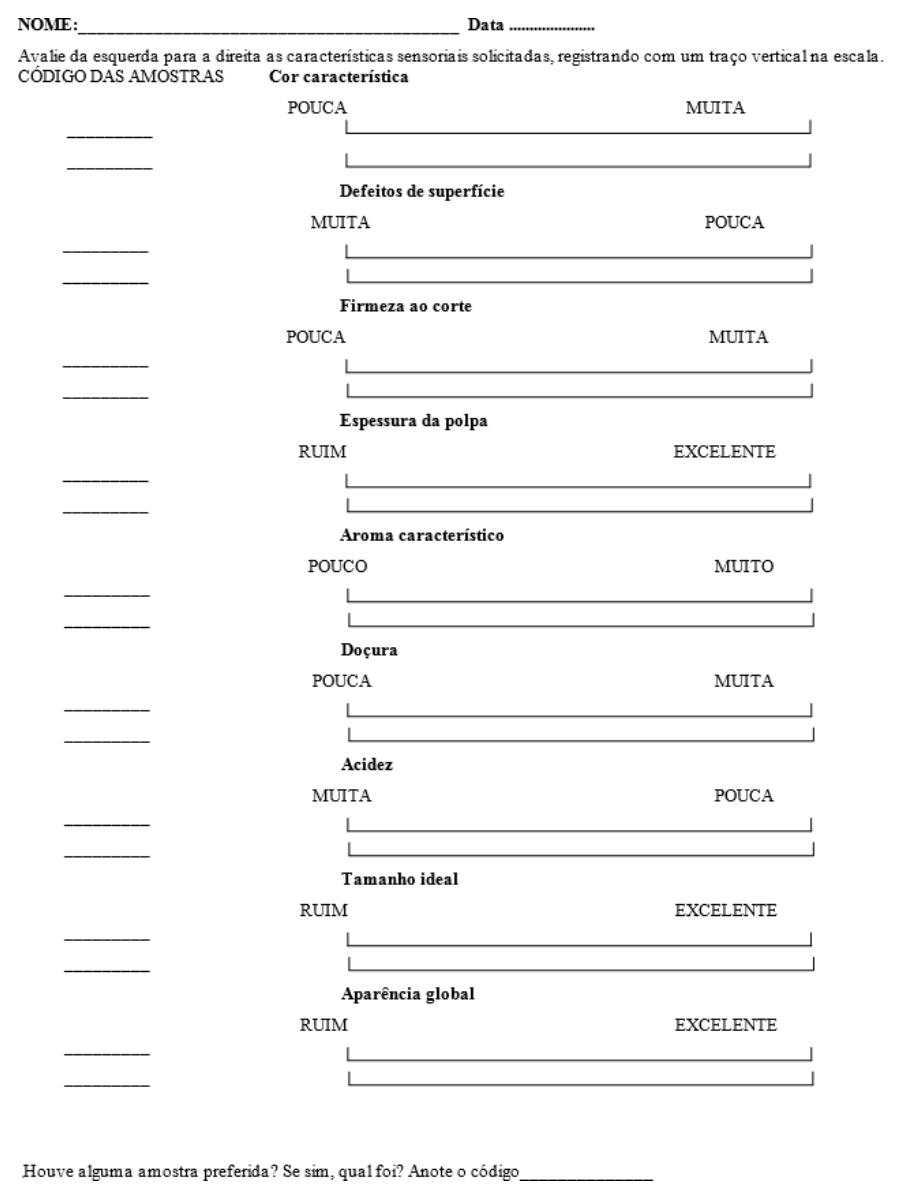

Figura 1. Modelo de ficha de análise sensorial descritiva. 
Foram selecionados 10 avaliadores experientes, de ambos os sexos, com faixa etária de 25 a 50 anos, fazendo parte da equipe, discentes e docentes da Universidade Federal de Pelotas. Os avaliadores foram orientados sobre as características do tomate, como: sabor (acidez e doçura), textura (firmeza ao corte e espessura da polpa) e coloração. Na Figura 1 é apresentado o modelo de ficha da análise descritiva $(A D)$.

Os termos e atributos que ancoraram essa avaliação foram: cor: $0=$ pouco e $9=$ muito, defeitos de superfície: $0=$ muito e $9=$ pouco, firmeza ao corte: $0=$ pouca e $9=$ muita, espessura da polpa: $0=$ ruim e $9=$ excelente, aroma característico: $0=$ pouco e $9=$ muito, doçura: $0=$ pouco e $9=$ muito, acidez: $0=$ muita e $9=$ pouco, tamanho ideal: $0=$ ruim e $9=$ excelente, aparência global: $0=$ ruim e $9=$ excelente.

\subsection{Análise estatística}

Os resultados foram submetidos à análise de variância ANOVA com comparação de médias através do teste de Tukey a um nível de significância de 5\%. Os gráficos tipo aranha apresentam resultados da analise sensorial.

\section{Resultados e Discussão}

Os resultados das análises de pH, sólidos solúveis e cor estão descritos na Tabela 1. Pode-se dizer que a coloração dos tomates é a característica mais importante, sendo por sua vez um fator importante na decisão de compra por parte do consumidor bem como um indicativo de maturação do fruto (LÓPEZ; GÓMEZ, 2004). A cor mais característica do tomate é o vermelho, sendo assim, entre as amostras, o acesso 14 foi o que obteve maior valor de $a^{\star}(27,0 \pm 5,1)$.

Tabela 1. Caracterização físico-química dos acessos de tomates orgânicos.

\begin{tabular}{|c|c|c|c|c|c|c|}
\hline \multirow[b]{2}{*}{ Tomates } & \multirow[b]{2}{*}{ Amostras } & \multirow[b]{2}{*}{$\mathrm{pH}$} & \multirow[b]{2}{*}{$\begin{array}{c}\text { SST } \\
\left({ }^{\circ} \text { Brix }\right)\end{array}$} & \multicolumn{3}{|c|}{ Cor } \\
\hline & & & & $\mathrm{L}^{*}$ & $a^{*}$ & $\mathrm{~b}^{*}$ \\
\hline \multirow{5}{*}{ Cereja } & $12 \mathrm{VD}$ & $3,5 \pm 0,1 c$ & $6,0 \pm 0,2 \mathrm{~cd}$ & $46,4 \pm 1,1 \mathrm{cb}$ & $-2,0 \pm 0,5 b$ & $25,4 \pm 3,0 \mathrm{c}$ \\
\hline & 13 & $3,4 \pm 0,1 c$ & $5,6 \pm 0,1 \mathrm{~cd}$ & $40,9 \pm 0,2$ de & $23,4 \pm 2,6^{a}$ & $15,7 \pm 0,3 d$ \\
\hline & 14 GVM & $4,3 \pm 0,0 \mathrm{a}$ & $8,1 \pm 0,3 \mathrm{a}$ & $44,0 \pm 0,5 \mathrm{~cd}$ & $27,0 \pm 5,1^{\mathrm{a}}$ & $19,5 \pm 1,5 d$ \\
\hline & $6 / 7$ & $3,9 \pm 0,0 b$ & $7,1 \pm 0,0 \mathrm{~b}$ & $40,6 \pm 0,3 e$ & $23,8 \pm 1,8^{\mathrm{a}}$ & $15,6 \pm 0,3 d$ \\
\hline & 4 GAM & $3,4 \pm 0,0 \mathrm{c}$ & $5,5 \pm 0,2 d$ & $50,8 \pm 2,0 a$ & $3,8 \pm 0,9 b$ & $39,5 \pm 1,5 a$ \\
\hline \multirow{2}{*}{ Salada } & 16 & $3,8 \pm 0,0 b$ & $4,2 \pm 0,4 e$ & $48,2 \pm 1,6 a b$ & $23,9 \pm 1,0 a$ & $33,1 \pm 2,0 b$ \\
\hline & 18 & $3,8 \pm 0,0 b$ & $6,2 \pm 0,0 \mathrm{c}$ & $44,4 \pm 1,3 \mathrm{~cd}$ & $22,6 \pm 0,4 a$ & $27,0 \pm 1,6 \mathrm{c}$ \\
\hline
\end{tabular}

Médias $(\mathrm{n}=3) \pm$ desvio padrão seguidas de letras distintas na coluna diferem entre si pelo teste de Tukey $(p<0,05)$. VD = Tomate tipo cereja verde, GVM = tomate tipo cereja garrafinha vermelho, GAM = tomate tipo cereja garrafinha amarelo. SST = Sólidos solúveis totais. 
O tomate do acesso 12 é o único que tem como característica a cor verde quando maduro, podendo ser observado pelo resultado do parâmetro $a^{*}(-2,0 \pm 0,5)$, já 0 acesso 4 tem como propriedade a cor amarela sendo indicada pelo valor de $b^{*}$ $(39,5 \pm 1,5)$. A amostra 18 foi a que obteve resultado menor para coloração vermelha. $O$ valor de $L^{*}$ corresponde à luminosidade do fruto, neste parâmetro praticamente não se obteve variação de valores.

Os valores de $\mathrm{pH}$ são semelhantes em todos os acessos, o valor mais elevado foi encontrado na amostra 14 (mais vermelho), $\mathrm{pH}=4,3 \pm 0,0$, e o mais baixo para amostra 4 (amarelo), $\mathrm{pH}=3,4 \pm 0,0$, mesmo valor encontrado por Rêgo, Finger e Casali (1999), que cita que $\mathrm{pH}$ de tomates com tonalidade vermelho forte são superiores aos frutos de casca amarela.

Os sólidos solúveis totais influenciam diretamente no sabor dos frutos. Pode-se notar que, algumas amostras não variaram significativamente entre si, entretanto a cultivar 14 foi a que apresentou valor mais alto de SST $(8,1 \pm 0,3)$, e a cultivar 16 a que apontou o menor valor $(4,2 \pm 0,4)$. Os valores encontrados estão dentro das faixas normais para tomates (PEDRO, 2004). No entanto, os valores mínimos de sólidos totais aceitos pelas indústrias de transformação de tomate não deve ser inferior a 4,0 ${ }^{\circ}$ Brix (SILVA; GIORDANO, 2013). Observou-se que todas as amostras de tomate, tem Brix superior a 4. Na Tabela 2 estão os resultados da caracterização dos compostos bioativos.

Tabela 2. Caracterização de compostos bioativos em tomates orgânicos.

\begin{tabular}{|c|c|c|c|c|c|}
\hline Tomate & Amostra & $\begin{array}{c}\text { DPPH } \\
\text { mg.TEACg } \\
\text { de ff }\end{array}$ & $\begin{array}{c}\text { Fenóis } \\
\text { mg.GAE.g }{ }^{-1} \\
\text { de ff }\end{array}$ & $\begin{array}{l}\text { Vitamina C } \\
\text { mg. } 100 \mathrm{~g}^{-1} \mathrm{ff}\end{array}$ & $\begin{array}{c}\text { Carotenoides } \\
\mu \mathrm{g} \cdot \mathrm{g}^{-1} \mathrm{ff}\end{array}$ \\
\hline \multirow{5}{*}{ Cereja } & $12 \mathrm{VD}$ & $4,5 \pm 0,1^{\mathrm{a}}$ & $1,5 \pm 0,0 \mathrm{c}$ & $1,5 \pm 0,0 \mathrm{e}$ & $18,8 \pm 0,0 b$ \\
\hline & 13 & $3,7 \pm 0,0 \mathrm{c}$ & $1,7 \pm 0,0 b$ & $0,9 \pm 0,0 \mathrm{f}$ & $17,4 \pm 0,0 \mathrm{c}$ \\
\hline & 14 GVM & $4,1 \pm 0,0 \mathrm{~b}$ & $1,8 \pm 0,0 b$ & $1,7 \pm 0,0$ de & $14,6 \pm 0,1 b$ \\
\hline & $6 / 7$ & $3,2 \pm 0,0 d$ & $1,7 \pm 0,0 b$ & $2,3 \pm 0,0 \mathrm{ab}$ & $19,3 \pm 0,0 \mathrm{a}$ \\
\hline & 4 GAM & $3,2 \pm 0,0 \mathrm{~d}$ & $2,1 \pm 0,0 \mathrm{a}$ & $2,5 \pm 0,1 \mathrm{a}$ & $18,5 \pm 0,3 b$ \\
\hline \multirow{2}{*}{ Salada } & 16 & $3,1 \pm 0,0 \mathrm{~d}$ & $1,8 \pm 0,0 b$ & $1,9 \pm 0,0 \mathrm{~cd}$ & $17,7 \pm 0,2 \mathrm{c}$ \\
\hline & 18 & $2,8 \pm 0,0 \mathrm{e}$ & $1,8 \pm 0,0 b$ & $2,1 \pm 0,1 b c$ & $18,8 \pm 0,1 b$ \\
\hline
\end{tabular}

Médias $(n=3) \pm$ desvio padrão seguidas de letras distintas na coluna diferem entre si pelo teste de Tukey $(p<0,05)$. VD = Tomate tipo cereja verde, GVM = tomate tipo cereja garrafinha vermelho, GAM = tomate tipo cereja garrafinha amarelo.

De acordo com os resultados obtidos pode-se observar que a amostra número 18 se destacou mais perante as outras, apresentando maior capacidade antioxidante $(2,8 \pm 0,0)$, o acesso 12 VD foi o que mostrou menor conteúdo $(4,5 \pm 0,1)$. Borguini (2006) encontrou resultados inferiores de atividade antioxidante em tomates cultivados em sistema convencional. Apesar da variação existente entre os acessos não houve diferenças significativas no teor de compostos fenólicos. A amostra que obteve maior média e por sua vez maior teor de compostos fenólicos foi o acesso 4 $(2,1 \pm 0,0)$. O teor de vitamina $C$ foi o componente que mais variou entre os parâmetros analisados, a amostra 13 apresentou valor bem inferior $(0,9 \pm 0,0)$ quando comparada as outras amostras, a amostra número 4 foi a que teve maior teor de 
ácido ascórbico $(2,5 \pm 0,1)$. Caris-Veyrat et al. (2004), observaram superioridade de $35 \%$ de vitamina C em tomates orgânicos quando comparado com tomates convencionais. O resultado mais elevado de carotenoides foi encontrado na amostra $6 / 7(19,3 \pm 0,0)$ e o menor valor na amostra $14(14,6 \pm 0,1)$. Sabe-se que a qualidade de um fruto na pós-colheita está diretamente relacionada com seu ponto de maturação, diversos trabalhos relatam que os compostos bioativos em geral são fortemente influenciados pelas práticas culturais (PAULA et al., 2015). O tomate é uma hortaliça com grande teor de carotenoides. Alguns carotenoides são precursores de vitamina $A$, uma função muito importante já que a sua deficiência pode acarretar problema de saúde pública. No tomate, o betacaroteno é o carotenoide pró-vitamina $A$ mais ativo, e que está associado à proteção de diversas doenças (CARVALHO et al., 2006). Considerando as evidências epidemiológicas, atualmente o tomate é considerado um alimento funcional, sendo responsável pela redução de diferentes tipos de doenças (BORGUINI, 2006).

Em relação à análise sensorial, foi utilizada a técnica de descrição sensorial através do método de análise descritiva, pois permite o levantamento, a descrição e a quantificação dos atributos detectáveis em um produto (ALCANTARA; FREITAS, 2018). A Figura 2 demonstra a média da avaliação sensorial de todos os tempos avaliados.

O primeiro grupo de tomates analisados foi o do tempo inicial, referente ao dia da colheita (Figura 2 - a). A amostra 13 apresentou maior valor para o atributo cor, a amostra com menor número de defeitos também foi o tomate do acesso 13. A amostra número 16 mostrou maior firmeza em relação ao corte, a amostra com melhor espessura de polpa foi a número 14 . Em relação ao aroma característico de tomate, a amostra 13 é a responsável por este atributo. A amostra que destacou maior teor de doçura foi a $6 / 7$ a amostra com maior teor de acidez foi a amostra número 4 . As melhores médias para as características de tamanho e aparência global foram obtidas pela amostra 13, as quais mais se aproximaram da avaliação máxima (9 pontos). Em relação aos resultados é possível verificar que o tomate número 13 foi o que alcançou melhores resultados quando comparado as outras amostras, a preferência pode ser explicada pelo fato da amostra ter cor vermelha característica e um tamanho padrão para tomates.

O segundo tempo avaliado (Figura 2 - b) perante análise sensorial foi o tempo oito, no qual os frutos foram armazenados em pratos cobertos com filme plástico em temperatura aproximadamente de $25 \pm 1{ }^{\circ} \mathrm{C}$. É possível analisar através do gráfico que as médias para cor de todas as amostras aumentaram consideravelmente. Segundo, Machado, Alvarenga e Florentino (2007) uma possível explicação para isso se dá ao fato que a temperatura é um fator ambiental significativo no desenvolvimento da cor do tomate, durante o amadurecimento temperaturas mais elevadas não inibem a síntese de betacaroteno, o qual é responsável por boa parte da cor dos frutos, intensificando a tonalidade do laranja para o vermelho. Em relação aos defeitos de superfície dos tomates, as médias de todas as amostras aumentaram significativamente, apontando para deterioração do mesmo. A amostra número 4 apresentou mais sinais de defeitos (rachaduras) do que as outras; a amostra que menos mudou quando comparada ao tempo inicial foi a amostra número 6/7. 

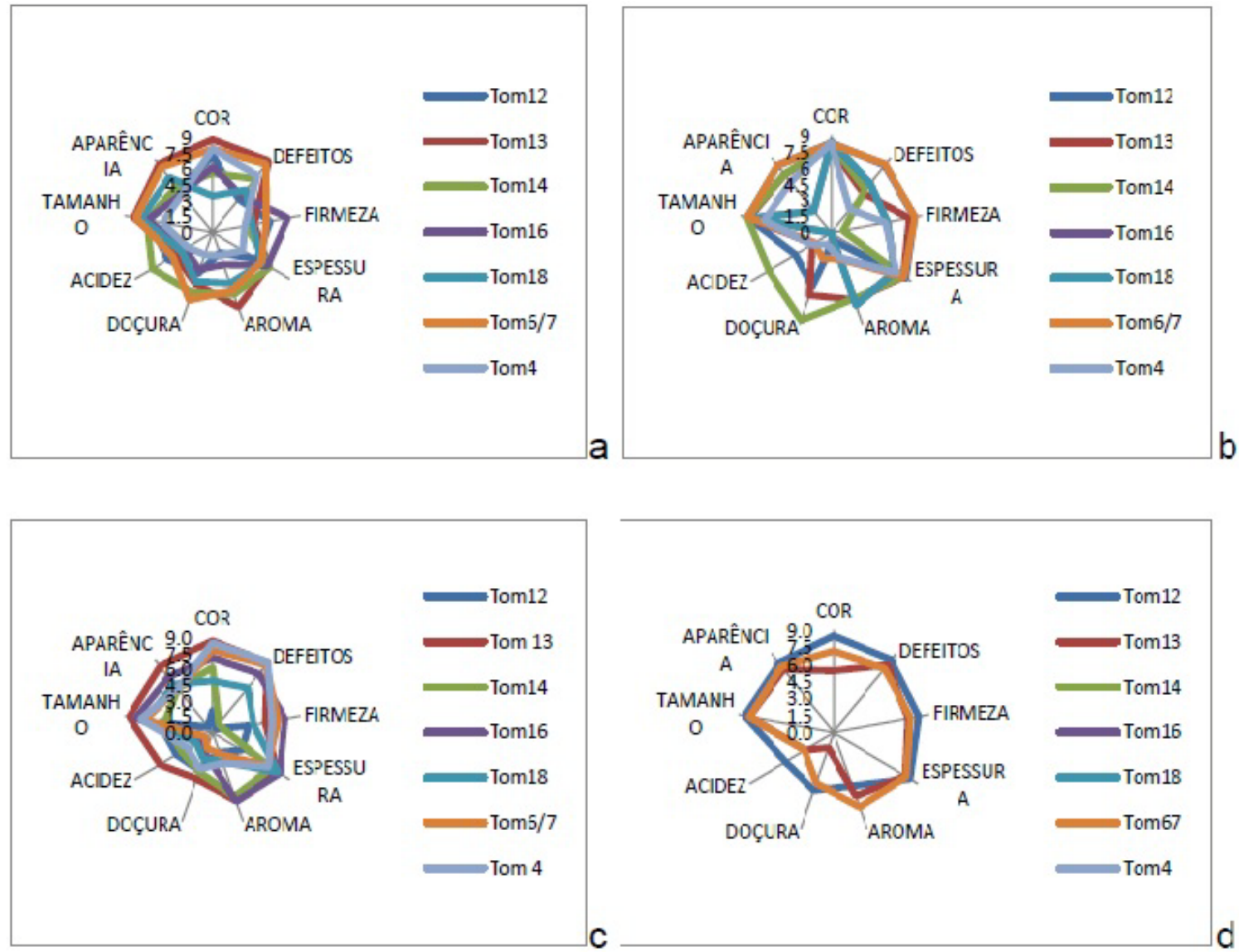

Figura 2. Gráfico aranha do perfil dos tomates no tempo zero (a), tempo oito temperatura ambiente (b), tempo oito refrigerado (c) e tempo doze refrigerado (d).

Quanto a firmeza dos frutos, pode-se dizer que a única amostra que sofreu alterações foi a número 14, que apresentou uma textura mais macia. Silva, Alvarenga e Maciel (2013) observaram que em tomate, a perda progressiva da firmeza com 0 amadurecimento é resultante da redução na espessura da parede celular e adesão entre as células adjacentes, devido a solubilização da protopectina na parede celular para formar pectina solúvel e outros produtos.

Todas as amostras tiveram um pequeno aumento na média em relação a espessura da polpa, a amostra 4 teve um aumento significativo em relação a este atributo. Ao comparar a amostra 13 do tempo inicial com a do tempo oito, o aroma característico diminuiu, entretanto em outras amostras houve um aumento na média. Quanto a doçura dos frutos, as amostras 12, 13, 14 apresentaram maiores valores para doçura, já as amostras 6/7 e 4 tiverem redução, ou seja, estavam menos doces. Os tomates das amostras 16 e 18 não estavam aptos para o consumo, pois apresentaram características de mofos, sendo assim não foi possível realizar avaliação. Em relação a acidez observou-se redução em todos os valores, sendo um indicativo de que todas as amostras não estavam com sabor ácido. Segundo estudado por Seleguini et al. (2009) essa redução é explicável pelo metabolismo climatérico do tomate, que faz com que os ácidos orgânicos sejam utilizados na respiração. Em relação ao tamanho e a aparência dos tomates analisados, as únicas amostras que apresentaram valores relacionados à insatisfatório, foram as amostras dos acessos 16 e 18 por conta da contaminação microbiológica na superfície. 
O terceiro tempo avaliado foi o tempo oito refrigerado a temperatura de $\pm 4{ }^{\circ} \mathrm{C}$ (Figura 2 - c) Em geral as colorações dos tomates refrigerados apresentaram-se inferiores aos resultados encontrados no tempo oito em temperatura ambiente, no qual a amostra 12 teve uma grande discrepância, observou-se grande descolaração. O tomate, em geral, apresenta sensibilidade ao frio, dependendo do estádio de amadurecimento em que se encontra. $O$ fruto está no grupo de produtos que sofrem danos com o frio (temperaturas abaixo de $7^{\circ} \mathrm{C}$ ), exigindo, portanto, maiores cuidados em ambientes refrigerados (CASTRO, 2003).

O tomate 14 e o tomate 12 apresentaram mais defeitos que as amostras do tempo oito em temperatura ambiente, entretanto a amostra 4 apresentou menos defeito (menor rachadura de superfície). Em relação a firmeza, as únicas amostras que apresentaram resultados superiores foram as amostras do tomate 16 e 4 . Todas as outras tiverem redução significativa em relação a espessura do fruto, com exceção da amostra 12 , que apresentou valores inferiores, indicando menor espessura da polpa, as demais amostras não variaram significativamente. No atributo aroma, as amostras concentraram mais os compostos voláteis, preservando as características de odor. Todas as amostras apresentaram valores menores tanto para doçura quanto para acidez. Diferente do tempo oito em temperatura ambiente as amostras 16 e 18 não indicaram a presença de mofo, sendo assim, foi possível realizar a avaliação sensorial de doçura e acidez, os valores não variaram em relação as outras amostras do mesmo período. O tamanho e aparência global do fruto foram semelhantes em algumas amostras. As amostras 16 e 18 apresentaram valores superiores para aparência global, provavelmente devido ao fato do não aparecimento de mofo. Já amostra número 12 obteve nota bem inferior para aparência global.

O quarto e último tempo analisado é o tempo doze refrigerado (Figura $2-\mathrm{d}$ ). Dentre todas as amostras, apenas os tomates, 12, 13 e 6/7 não estavam deteriorados. Em relação a cor dos frutos a amostra 12 apresentou coloração mais intensa do que o tempo anterior, as demais amostras mantiveram-se semelhantes aos resultados anteriores. Para o atributo defeitos de superfície, a amostra 12 também apresentou resultados superiores, as amostras 13 e 6/7 continuaram com resultados próximos aos já citados anteriormente. Houve um pequeno acréscimo na média para a característica de firmeza ao corte nas três amostras. A espessura se manteve praticamente a mesma para as amostras 6/7 e 13, a amostra 12 apresentou valor superior ao encontrado no tempo oito refrigerado. $\mathrm{O}$ aroma diminuiu para a amostra 12 , as demais tiveram um pequeno aumento. A doçura aumentou para amostra 12 e 6/7 consideravelmente, entretanto as mesmas obtiveram valores maiores para acidez. A amostra 13 perdeu acidez e doçura, quando comparada com os tempos analisados anteriormente. Os resultados para tamanho característico do fruto não variaram. Já a aparência global, aumentou para a amostra 6/7 e 12, a amostra 13 recebeu valor inferior. Como se esperava, o uso da refrigeração aumenta a vida útil de praticamente todos os alimentos, o emprego da refrigeração nos acessos de tomates foi apontado como um fator positivo, entretanto o uso de temperaturas muito baixas, como Castro (2003) havia mencionado em sua pesquisa, não é adequado para o armazenamento do tomate.

\section{Conclusão}

Os tomates avaliados este estudo, cultivados pela Embrapa Terras Baixas, possuem quantidade de compostos bioativos benéficas à saúde. Os acessos 14 e 6/7 se 
mostraram mais adocicados, o acesso 6/7 também apresentou maior conteúdo de vitamina C. Em geral a coloração da casca das amostras é de vermelho-alaranjada, com exceção dos acessos que possuem como característica coloração amarela-verde. A refrigeração concedeu maior tempo de vida útil a algumas amostras, entretanto, não se indicam temperaturas inferiores a $7^{\circ} \mathrm{C}$ para o armazenamento dos frutos. $\mathrm{O}$ estudo indica que os acessos pesquisados podem ser produzidos para uma futura comercialização.

\section{Referências}

ALCANTARA, M.; FREITAS-SÁ, D. G. C. Metodologias sensoriais descritivas mais rápidas e versáteis - uma atualidade na ciência sensorial. Brazilian Journal of Food Technology. Campinas, v. 21, e2016179, 2018. http://dx.doi.org/10.1590/19816723.17916.

BORGUINI R. G. Avaliação antioxidante e de algumas características físicoquímicas do tomate orgânico em comparação ao convencional. 178f. 2006. Tese (Doutorado em Saúde Publica. Faculdade de Saúde Pública. Universidade de São Paulo, São Paulo. 2006.

BRAND-WILLIAMS, W.; CUVELIER, M.E.; BERSET, C. Use of a free radical method to evaluate antioxidant activity. LWT Food Science and Technology, v. 28, n. 1, p. 2530, 1995. https://doi.org/10.1016/S0023-6438(95)80008-5.

CARIS-VEYRAT, C. et al. Influence of organic versus conventional agricultural. Journal of Agricultural Food Chemistry, v. 52, n. 21, p.6503-6509, 2004. https://doi.org/10.1021/jf0346861.

CARVALHO, P. G. B. et al. Hortaliças como alimentos funcionais. Horticultura Brasileira, Brasília, v. 24, n. 4, p. 147-152, 2006. http://dx.doi.org/10.1590/S010205362006000400001.

CASTRO, V.A.S.P.T. Controle do amadurecimento pós-collheita do tomate "Carmem" tratado com ácido 2-cloroetil fosfônico. 88f. 2003. Dissertação (Mestrado Tecnologia Pós-Colheita) Universidade Federal de Campinas, Faculdade de Engenharia Agrícola, Campinas. 2003.

COSTA, E. S. et al. Avaliação de substratos alternativos para a produção de mudas de tomateiro no agreste alagoano. Horticultura Brasileira, v. 28, n. 2, p.675-682, 2010. http://dx.doi.org/10.1590/S0034-737X2013000500011.

GIULIO G. Setor tomateiro cresce e demanda aumento de pesquisas. Inovação Uniemp, v. 3, n.1, p.42-44, $2007 . \quad$ Disponível em: $<$ http://inovacao.scielo.br/scielo.php?script=sci_arttext\&pid=S1808$23942007000100023 \&$ lng=es\&nrm=is> Acesso 28 dez. 2020.

GULARTE, M. A. Manual de análise sensorial de alimentos. Pelotas: UFPel, 2009. 106p.

Instituto Brasileiro de Geografia e Estatística. IBGE Censo Agropecuário. 2017.

LÓPEZ C. A. F., GÓMEZ P.A. Comparison of color indexes for tomato ripening. Horticultura Brasileira, v.22, n.3, p.534-537, 2004. https://doi.org/10.1590/S010205362004000300006. 
MACHADO A. Q.; ALVARENGA M. A. R; FLORENTINO C. E. T. Produção de tomate italiano (saladete) sob diferentes densidades de plantio e sistemas de poda visando ao consumo in natura. Horticultura Brasileira, v. 25, p.149-153, 2007. https://doi.org/10.1590/S0102-05362007000200004.

MAPA - MINISTÉRIO DA AGRICULTURA, PECUÁRIA E ABASTECIMENTO. Instrução normativa ${ }^{\circ}$ 46, de 6 de Outubro de 2011.

NOGUEIRA R. B.; ROSADO P. L.; GOMES A. S. Determinantes da demanda de hortaliças orgânicas em ilhéus. Conjuntura \& Planejamento, Salvador, n.162, p.6671, $2009 . \quad$ Disponível em: <http://www.sei.ba.gov.br/site/publicacoes/sumarios/c\&p162/c\&p162_pag_66.pdf>. Acesso 28 dez. 2020.

PAULA, J.T. et al. Características físico-químicas e compostos bioativos em frutos de tomateiro colhidos em diferentes estádios de maturação. Horticultura Brasileira, v. 33, p. 434-440, 2015. http://dx.doi.org/10.1590/S0102-053620150000400005.

PEDRO, A. M. K. Determinação simultânea e não-destrutiva de sólidos totais e solúveis, licopeno e betacaroteno em produtos de tomate por espectroscopia no infravermelho próximo utilizando calibração multivariada. 102f. 2004. Dissertação (Mestrado em Físico-Química), Instituto de Química, UNICAMP, Campinas. 2004.

RÊGO R.; FINGER L.; CASALI W. D. Qualidade de frutos de tomate da cv. Santa Clara, mutante de fruto amarelo e seus híbridos F1. Horticultura Brasileira, v. 17, n. 2, p.106-109, 1999. https://doi.org/10.1590/S0102-05361999000200006

RODRIGUEZ-AMAYA, D. B. A guide to carotenoid analysis in foods. Washington: Internacional Life Sciences Institute Press. 64p, 2001.

SELEGUINI A. et al. Vida útil e qualidade de frutos colhidos de plantas de tomateiro tratadas com paclobutrazol. Horticultura Brasileira, v. 27, p. S3717-S3723, 2009. http://dx.doi.org/10.1590/S0034-737X2011000400010.

SILVA, E. C.; ALVARENGA, P. P. M.; MACIEL, G.M. Avaliações físico-químicas de frutos de tomateiro em função de doses de potássio e nitrogênio. Bioscience Journal, v. 29, n. $6, \quad$ p. 1788-1795, 2013. Disponível em: $<$ http://www.seer.ufu.br/index.php/biosciencejournal/article/view/21896>. Acesso 28 dez. 2020.

SILVA, J.B.C.; GIORDANO, L.B. Tomate para processamento industrial. Brasília: Embrapa Comunicação para Transferência de Tecnologia - Embrapa Hortaliças, 168p. 2013.

SINGLETON, V.L.; ROSSI, J.A.JR. Colorimetry of total phenolics with phosphomolybdic - phosphotungstic acid reagents. American Journal of Enologyand Viticulture, v.16, p.144-158, $1965 . \quad$ Disponível em: <https://www.ajevonline.org/content/16/3/144>. Acesso 28 dez. 2020.

VALENT, J. Z. et al. Qualidade de produtos orgânicos: a percepção dos produtores de hortaliças de uma feira ecológica em Porto Alegre - RS. Revista Eletrônica em Gestão, Educação e Tecnologia Ambiental - REGET, v. 18, p.1072-1082, set./dez. 2014. http://dx.doi.org/10.5902/2236117013839. 
VINCl, G. et al. Ascorbic acid in exotic fruits: a liquid chromatographic investigation. Food Chemistry, v.53, p.211-214, 1995. https://doi.org/10.1016/0308-8146(95)907915.

\section{Autores}

Eduarda Caetano Peixoto ${ }^{1, \star}$, Bianca Pio Ávila², Irajá Ferreira Antunes ${ }^{3}$, Márcia Arocha Gularte $^{4}$

1. Curso de Pós-graduação Lato Sensu em Ciência dos Alimentos, Centro de Ciências Químicas, Farmacêuticas e de Alimentos, Universidade Federal de Pelotas, Campus Universitário, Caixa Postal, 354, 96010-900, Pelotas, RS, Brasil.

2. Universidade Federal de Pelotas, Departamento de Ciência e Tecnologia de Alimentos. Campus Universitário, Caixa Postal, 354, 96010-900, Pelotas, RS, Brasil.

3. Embrapa Clima Temperado, Rodovia BR-392, Km 78, $9^{\circ}$ Distrito, Monte Bonito, Caixa Postal, 403, 96010-971, Pelotas, RS, Brasil.

4. Centro de Ciências Químicas, Farmacêuticas e de Alimentos, Universidade Federal de Pelotas, Campus Universitário, Caixa Postal, 354, 96010-900, Pelotas, RS, Brasil.

*Autor para correspondência: eduardacpeixoto@hotmail.com 